\title{
Molekulare Allergologie - was bringt sie für den Patienten?
}

A llergien sind auf dem Vormarsch und nehmen epidemische Ausmaße an - das ist mittlerweile auch in der Politik erkannt worden. Foren auf dem gerade vergangenen 4. Gemeinsamen Deutschen Allergiekongress wie „Allergien und Verbraucherschutz“ oder „Aktionsplan gegen Allergien“ unter Beteiligung des Bundesministeriums für Ernährung, Landwirtschaft und Verbraucherschutz unterstreichen den erforderlichen Handlungsbedarf. Forschungsaktivitäten und auch der daraus resultierende Wissenszuwachs haben zum Glück in den letzten Jahren auch in Deutschland rasch zugenommen. Der Kongress in Berlin unter dem Motto „Allergologie in der Mitte Europas“ machte dies mehr als deutlich. Eine ausführliche Berichterstattung finden Sie in diesem Heft ab Seite 488.

Da das Spektrum der Auslöser von allergischen Reaktionen sehr komplex ist, besteht nach wie vor berechtigtes Interesse, die Auslöser zu erkennen und zu detektieren. Durch Veränderungen in unserer Umwelt, etwa durch den Klimawandel, in unserer Arbeitswelt, wie durch die Einführung neuer Technologien, und Veränderungen im Lebensstil können neue Allergenquellen und Belastungssituationen auftreten. Wissenszuwachs auf diesem Gebiet muss dem Verbraucher, dem Beschäftigten und natürlich auch dem betroffenen Allergiker zugutekommen. Der Beitrag zum aktuellen Stand der berufsbedingten Typ-I-Allergien in diesem Heft (S. 538) beschäftigt sich daher mit Berufskrankheiten allergischer Genese, ihren unterschiedlichen Auslösern sowie den Entwicklungen zur Standardisierung der entsprechenden Diagnostik und der Allergenquantifizierung zur Expositionsüberwachung am Arbeitsplatz. Dies ist ein wichtiger Schritt zur Primärprävention.

Mittlerweile sind wir im Zeitalter der molekularen Allergologie angekommen. Spannende Erkenntnisse und mehrdimensionales Denken hinsichtlich Proteincharakterisierung, Aufdeckung struktureller „Nur in Kooperation zwischen Verwandtschaften von Allgrundlagenorientierten Bio- ergenmolekülen sowie wissenschaftlern und klini- Kreuzreaktivitäten, die reschen Kollegen kann der Weg kombinante Herstellung ,vom Epitop bis zum Patienten" von Allergenen und mehr beschritten werden." finden zum Teil schon

Einzug in eine komponentenaufgelöste Diagnostik. Durch Allergenchips kann in einem Screening schnell ein Allergogramm, also ein patientenspezifischer Fingerabdruck, erstellt werden, welcher zeigt, gegen welche Proteine IgE-Reaktivitäten ausgebildet wurden und welche damit klinisch relevant sein können. Allerdings steht die Frage im Raum: Was bringt das für den Patienten? Nur in Kooperation zwischen grundlagen- und mechanismenorientierten Biowissenschaftlern und klinischen Kollegen kann der Weg „vom Epitop bis zum Patienten“ beschritten werden, so dass die Erkenntnisse der molekularen Allergologie in thera-

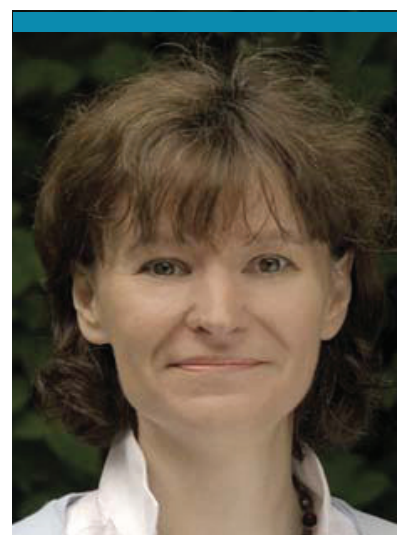

Prof. Dr. Monika Raulf-Heimsoth, Kompetenz-Zentrum Allergologie/Immunologie, BGFA - Forschungsinstitut für Arbeitsmedizin der Deutschen Gesetzlichen Unfallversicherung, Ruhr-Universität, Bochum

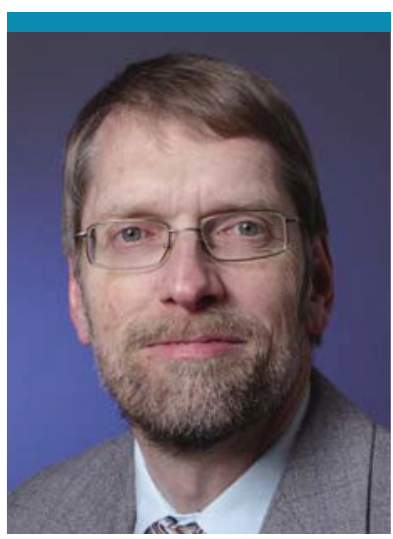

Prof. Dr. Arnd Petersen, Molekulare und klinische Allergologie, Forschungszentrum Borstel peutische Ansätze münden können. Denn nur eine Diagnostik, die zu therapeutischen Konsequenzen führen kann, ist sinnvoll. Der Artikel in diesem Heft ab Seite 551 fasst die Erkenntnisse zur molekularen Allergenstruktur zusammen.

Beide Beiträge sind Herrn Dr. Wolf-Meinhard Becker, Forschungszentrum Borstel, zum 65. Geburtstag gewidmet, der sich mehr als 25 Jahre seines Forscherlebens mit der Allergenidentifizierung und -aufklärung sowie der Interaktion der Allergene mit dem Immunsystem an den Grenzflächen des Organismus erfolgreich beschäftigt hat.

Für den klinisch arbeitenden Allergologen hält dieses Heft außerdem noch die Aktualisierung der Leitlinie der allergologischen Fachgesellschaften zur spezifischen Immuntherapie bereit (Seite 508). Auch hier zeigt sich der Wissenszuwachs in der Allergologie, denn im Vergleich zur alten Fassung aus dem Jahr 2006 hat der Umfang um elf auf jetzt insgesamt 30 Druckseiten zugenommen.

Wir sind sicher, dass für jeden etwas Informatives dabei ist und der Blick über den eigenen Tellerrand gelingt, so dass die Lektüre für Sie von Gewinn sein wird - das zumindest wünschen wir uns.

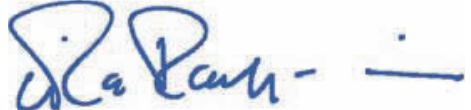

Prof. Dr. Monika Raulf-Heimsoth

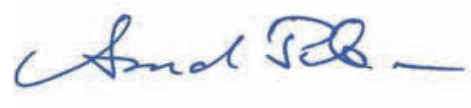

Prof. Dr. Arnd Petersen 